\title{
Acute myocardial infarctions and stroke triggered by laboratory-confirmed respiratory infections in Denmark, 2010 to 2016
}

Jessica Ohland ${ }^{1}$, Charlotte Warren-Gash ${ }^{2}$, Ruth Blackburn ${ }^{3}$, Kåre Mølbak ${ }^{1,4}$, Palle Valentiner-Branth ${ }^{1}$, Jens Nielsen ${ }^{1}$, HanneDorthe Emborg ${ }^{1}$

1. Statens Serum Institut, Copenhagen, Denmark

2. Faculty of Epidemiology \& Population Health, London School of Hygiene \& Tropical Medicine, London, United Kingdom

3. Institute of Health Informatics, University College London, London, United Kingdom

4. Institute of Veterinary and Animal Sciences, Faculty of Health and Medical Sciences, University of Copenhagen, Denmark

Correspondence: Hanne-Dorthe Emborg (hde@ssi.dk)

Citation style for this article:

Ohland Jessica, Warren-Gash Charlotte, Blackburn Ruth, Mølbak Kåre, Valentiner-Branth Palle, Nielsen Jens, Emborg Hanne-Dorthe . Acute myocardial infarctions and stroke triggered by laboratory-confirmed respiratory infections in Denmark, 2010 to 2016. Euro Surveill. 2020;25(17):pii=1900199. https://doi. org/10.2807/1560-7917.ES.2020.25.17.1900199

Article submitted on 21 Mar 2019 / accepted on 03 Mar 2020 / published on 30 Apr 2020

Background: Several studies have investigated a possible association between respiratory infection and acute myocardial infarction (MI). As both influenza and pneumococcal infections are vaccine preventable, understanding the populations affected by virusinduced cardiovascular complications is important to guide public health and clinical practice. Aim: This observational study aimed to quantify the association between laboratory-confirmed respiratory bacteria or virus infections and risk of first $M I$ or stroke, by using self-controlled case series (SCCS) analysis of anonymised linked electronic Danish health records.

Methods: The SCCS method was used to determine the relative incidence of the first event of $\mathrm{MI}$ and stroke occurring within $\mathbf{2 8}$ days after laboratory-confirmed respiratory infections compared with the baseline time period. Results: In the age and season adjusted analyses for first acute MI, the incidence ratios (IR) of a MI event occurring during the risk period were significantly elevated following a Streptococcus pneumoniae infection with values of $20.1,11.0$ and 4.9 during 1-3, 4-7 and 8-14 days, respectively and following respiratory virus infection with values of 15.2, 4.5 and 4.4 during 1-3, 8-14 and 15-28 days, respectively. The significantly elevated IRs for stroke following an $S$. pneumoniae infection were 25.5 and 6.3 during 1-3 and 8-14 days, respectively and following respiratory virus infection $8.3,7.8$ and 6.2 during $1-3,4-7$ and 8-14 days, respectively. Conclusion: This study suggested a significant cardiovascular event triggering effect following infection with $S$. pneumoniae and respiratory viruses (mainly influenza), indicating the importance of protection against vaccine-preventable respiratory infections.

\section{Introduction}

The leading causes of death worldwide for the past 15 years were ischaemic heart disease and stroke [1], which is also the case in Denmark ( http://www.healthdata.org/denmark). An association between circulation of seasonal influenza and acute cardiovascular events was first suggested in the 1930s, although these findings were limited by risk of ecological bias and lack of control for potential confounding factors [2]. A systematic review from 2009 found that influenza, mostly defined by clinical symptoms alone, appeared to trigger acute myocardial infarction and death from cardiovascular disease [3] and a meta-analysis of case-control studies from 2015 also found a significant association between recent respiratory infection and acute myocardial infarction [4]. Kwong et al. [5] used a laboratoryconfirmed influenza virus infection as exposure and found an association with acute myocardial infarction in a Canadian population.

Whether other respiratory organisms might act as triggers for cardiovascular events was investigated in a time series analysis using population-level data from England. This study showed a significant association between the timing of different laboratory-confirmed respiratory viral infections and myocardial infarction and ischaemic stroke hospitalisations among older people. The respiratory viruses investigated included adenovirus, human metapneumovirus, influenza virus, respiratory syncytial virus and rhinovirus [6]. These findings were supported by self-controlled case-series analyses of Scottish individuals, in which incidence ratios (IR) for myocardial infarction and stroke were significantly raised following laboratoryconfirmed Streptococcus pneumoniae and influenza virus infections, and raised point estimates were also observed following other laboratory-confirmed 


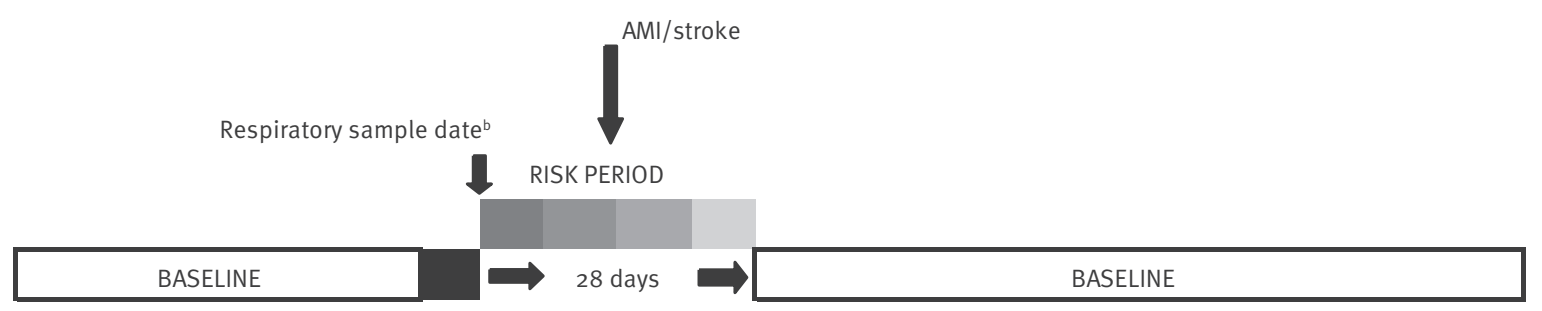

1 January 2010

31 December 2016

STUDY PERIOD BEGINS

STUDY PERIOD ENDS

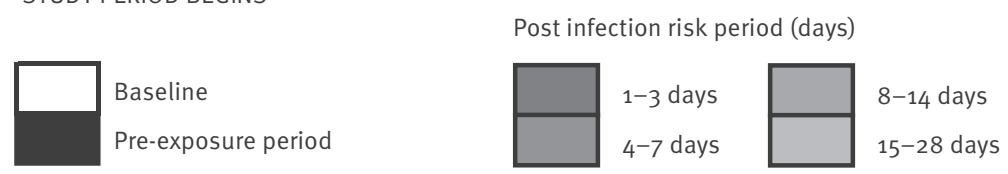

AMI: acute myocardial infarction.

${ }^{a}$ Modified after Petersen et al. [12].

${ }^{\mathrm{b}}$ The date when the sample is taken approximates the date of symptom onset of a respiratory infection.

respiratory virus infections [7]. A systematic review and meta-analysis of observational studies concluded that major cardiac complications occurred in a substantial proportion of the patients with community-acquired pneumonia, which can be caused by a range of pathogens [8]. Currently both influenza virus and pneumococcal infections are vaccine preventable and several vaccines against respiratory syncytial virus are in the pipeline [9]. However influenza and pneumococcal vaccine uptake is sub-optimal across much of Europe. Understanding the relative effects of different organisms on cardiovascular complications as well as the populations affected, will help to inform research into interventions and guide prevention strategies.

Denmark has well-functioning national electronic health registries, including the microbiology database $(\mathrm{MiBa})$ which allows real-time sharing of microbiological test results nationwide and integrates into the national eHealth infrastructure [10]. We aimed to test whether previous findings on cardiovascular events triggering effects of laboratory-confirmed respiratory pathogens also applied to a different northern European population than that of England and Scotland. The aim of our study was to quantify the association between laboratory-confirmed respiratory bacteria or virus infections and risk of first myocardial infarction or stroke using self-controlled case series (SCCS) analysis of anonymised linked electronic health records from Denmark.

\section{Methods}

\section{Study design}

We used the SCCS method to estimate the relative incidence of myocardial infarction and stroke following laboratory-confirmed respiratory infections compared with baseline time periods [11] - Figure 1. The SCCS method is best suited for acute events and transient exposures [12]. Only individuals who have a record of exposure (diagnosed with a respiratory infection) and an outcome (myocardial infarction or stroke) were included. The comparisons were made within individuals, implying that factors constant over time cancel out, i.e. that there is implicit control for fixed confounding factors. Factors that varied over time such as age and season were accounted for in the analysis. The study period was 1 January 2010 to 31 December 2016.

Following the time of a respiratory infection symptom onset, which was approximated by the date a sample had been taken for laboratory confirmation, a 28 day risk period was considered. Time outside the 28 day window was counted as baseline. The 28 day risk period was further divided into four periods, 1 to 3 days, 4 to 7 , 8 to 14 and 15 to 28 days (Figure 1). These periods were chosen for the purpose of direct comparison with other studies. Fourteen days before and the day of sampling were considered as pre-exposure period and excluded from the baseline period, because being diagnosed with an acute myocardial infarction or stroke during this time may change the likelihood of being sampled and diagnosed with a respiratory infection. The SCCS methods compares the likelihood of an acute myocardial infarction or stroke during the risk periods (after a laboratory-confirmed respiratory infection) to the likelihood outside the risk periods (baseline) within a person. Comparing the incidence risk between two time periods gives a relative incidence risk. 
Individuals included in the study, Denmark, 1 January 2010-31 December 2016 ( $\mathrm{n}=1,350$ individuals)

A. Myocardial infarction

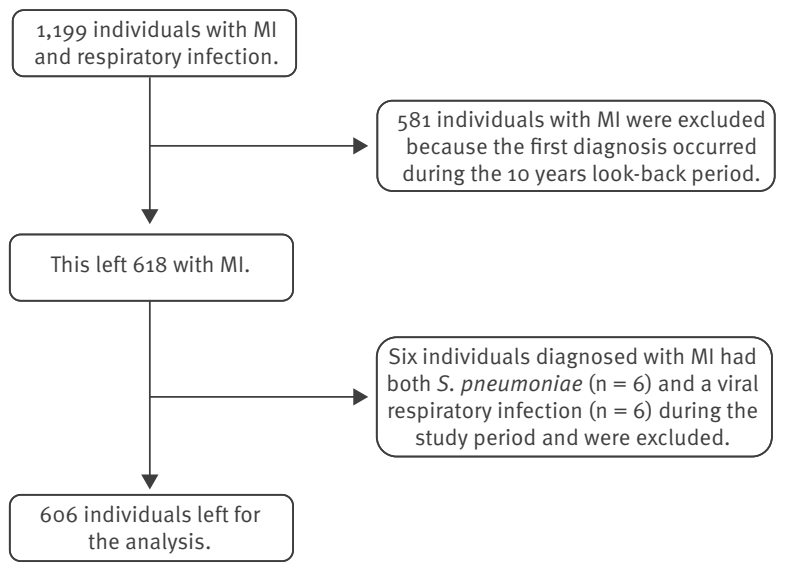

\section{B. Stroke}

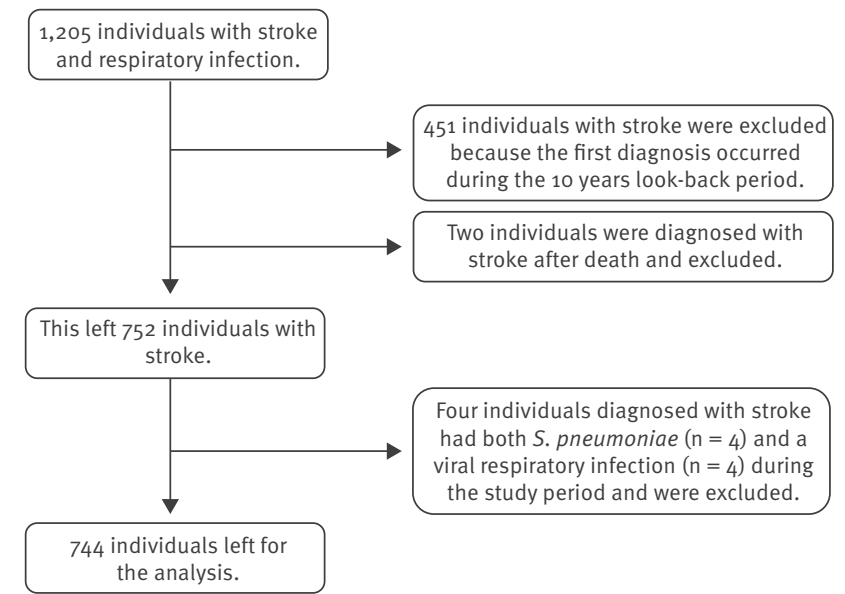

MI: myocardial infection; S. pneumoniae: Streptococcus pneumoniae.

Ten individuals, six with MI and four with stroke, had a dual viral-bacterial infection. Each of them appeared on the two data files with viral and bacterial pathogens simultaneously, so their numbers were excluded twice.

\section{Data sources}

The Civil Registration System

The Danish Civil Registration System (CRS) was established on 1 April 1968 and since then a unique personal identification number has been assigned to all Danish residents. CRS contains continuously updated information on vital status and permanent residence in Denmark. From CRS, information on birthdate, sex and vital status were extracted.

\section{Danish National Patient Register}

The Danish National Patient Register (DNPR) contains information on all hospital admissions and outpatient treatments in Denmark [13]. Date of admission and discharge are available for each registration in DNPR. We used date of admission as date of diagnosis for the acute myocardial infarction or stroke. All disease diagnoses are classified according to World Health International Statistical Classification of Diseases and Health-related Problems, $10^{\text {th }}$ Revision (ICD-10) [14].

Danish Microbiology database

The Danish Microbiology database (MiBa) was initiated in January 2010 and includes all microbiological test results from all the departments of clinical microbiology in Denmark. Each registration contains information on sample date, laboratory analysis and test result [10]. Both records of respiratory virus infections and invasive pneumococcal disease infections were extracted from MiBa. Individuals could be swabbed at a general practitioner's office, or at a hospital.
Pneumococcal laboratory database at Statens Serum Institut

In Denmark, the national surveillance of invasive pneumococcal disease (IPD) has been centralised at Statens Serum Institut (SSI) since 1938. All clinical microbiology departments in Denmark submit IPD isolates for serotype determination to the National Neisseria and Streptococcus Reference Laboratory (NSR) at SSI.

\section{Data extraction and linking}

All the above registries contain patients' information under their respective unique personal identification number from CRS, which can be used for record linkage as described below.

Hospital admissions for first acute myocardial infarction (ICD-10 codes: 121 and I23) and/or stroke (ICD-10 codes: 160, 161 and 163) in individuals aged 40 years and above during the study period 1 January 2010 to 31 December 2016 were extracted from the DNPR. In the SCCS method, repeated events must be independent. The risk of a subsequent event may change after experiencing either a myocardial infarction or stoke. We therefore applied a 10 year look back from 1 January 2000 to 31 December 2009 to identify individuals who had hospital admissions for acute myocardial infarction or stroke before the study period.

Specimens testing positive for any of the following viruses from 1 January 2010 to 31 December 2016 were extracted from MiBa: human metapneumovirus, influenza virus, parainfluenza virus, respiratory syncytial virus, or rhinovirus. During the same time period, specimens testing positive for $S$. pneumoniae were extracted from MiBa and the pneumococcal database 
Descriptive statistics of individuals $\geq 40$ years old with first myocardial infarction or stroke and at least one respiratory infection, Denmark, 1 January 2010-31 December $2016(n=1,350)$

\begin{tabular}{|c|c|c|c|c|}
\hline \multirow{2}{*}{ Characteristics } & \multicolumn{2}{|c|}{ Myocardial infarction } & \multicolumn{2}{|c|}{ Stroke } \\
\hline & $\mathrm{n}$ & $\%$ & $\mathrm{n}$ & $\%$ \\
\hline \multicolumn{5}{|l|}{ Sex } \\
\hline Female & 253 & 41.7 & 347 & 46.6 \\
\hline \multicolumn{5}{|l|}{ Age group in years at first cardiovascular event } \\
\hline $40-49$ & 40 & 6.6 & 35 & 4.7 \\
\hline $50-59$ & 95 & 15.7 & 91 & 12.2 \\
\hline $60-69$ & 156 & 25.7 & 211 & 28.4 \\
\hline $70-79$ & 175 & 28.9 & 216 & 29.0 \\
\hline $80-89$ & 121 & 20.0 & 152 & 20.4 \\
\hline$\geq 90$ & 19 & 3.1 & 39 & 5.2 \\
\hline Total & 606 & 100 & 744 & 100 \\
\hline \multicolumn{5}{|l|}{ Stratified age in years at first cardiovascular event } \\
\hline $40-64$ & 210 & 34.7 & 214 & 28.8 \\
\hline$\geq 65$ & 396 & 65.3 & 530 & 71.2 \\
\hline Total & 606 & 100 & 744 & 100 \\
\hline \multicolumn{5}{|l|}{ Mortality } \\
\hline Mortality $\leq 30$ days after cardiovascular event & 51 & 8.4 & 88 & 11.8 \\
\hline Mortality during study period & 242 & 39.9 & 336 & 45.2 \\
\hline Total & 606 & 100 & 744 & 100 \\
\hline \multicolumn{5}{|c|}{ Laboratory-confirmed respiratory infection episodes ${ }^{a, b}$} \\
\hline Streptococcus pneumoniae & 222 & 35.4 & 332 & 43.3 \\
\hline Respiratory virus ${ }^{\mathrm{c}}$ & 406 & 64.6 & 434 & 56.7 \\
\hline Total & 628 & 100 & 766 & 100 \\
\hline
\end{tabular}

a Myocardial infarction individuals and multiple samples: one individual has four viral samples, one individual has three viral samples and 12 individuals have two viral samples. Five individuals have two bacterial samples.

${ }^{\text {b }}$ Stroke individuals and multiple samples: three individuals have three viral samples, six individuals have two viral samples. One individual has three bacterial samples and eight individuals have two bacterial samples.

${ }^{\mathrm{c}}$ Human metapneumovirus, influenza virus, parainfluenza virus, respiratory syncytial virus, or rhinovirus.

at SSI. For each individual, laboratory detection of the same virus infection or pneumococcal infection within 28 days was considered the same infection, and only the earliest infection was considered for this study.

We used deterministic linkage to link data from DNPR to MiBa data and the pneumococcal laboratory data through the unique personal identification number. Only individuals aged 40 years and above and who were diagnosed with both an event (myocardial infarction or stroke) and with at least one of the respiratory infections during the study period were included in the study. Vital status from the CRS registry was linked to the data to identify individuals who died at any point during the study period. Individuals could enter the study on 1 January 2010 or when they reached 40 years of age after that date and exit the study at death or at 31 December 2016. The 40 year lower age limit for inclusion in the study was chosen for comparability to other studies using a similar approach $[6,7]$.

\section{Statistical analysis}

We investigated the relative incidence of a first myocardial infarction or stroke occurring within 28 days after the beginning of laboratory-confirmed respiratory infections with S. pneumoniae, human metapneumovirus, influenza virus, parainfluenza virus, respiratory syncytial virus, or rhinovirus as compared with the baseline time period for each individual using conditional Poisson regression.

The time dependent variables such as age and season are not accounted for in the basic SCCS method, but can be included in the model [11]. Age was controlled for in 5-year bands $(40-44,45-49,50-54$, $55-59,60-64,65-69,70-74,75-79,80-84,85-89$, 90-94, 95-99,100-104). Seasons were categorised as 3-month bands, repeated to span the study period (Jan-Mar, Apr-Jun, Jul-Sep, Oct-Dec).

Individuals experiencing acute myocardial infarction or stroke have an increased risk of dying. In the SCCS method, experiencing the outcome event should not censor follow-up, but this assumption can be violated for fatal cardiovascular events, which may lead to bias in either direction [12]. We therefore performed a sensitivity analysis, where individuals who died within 30 days following an acute myocardial infarction or stroke were excluded from the analysis to investigate this potential bias. 
Age and season adjusted incidence ratio for first acute myocardial infarction $(\mathrm{n}=606$ individuals) and first stroke ( $\mathrm{n}=744$ individuals) in periods after Streptococcus pneumoniae, respiratory viruses (combined) and influenza infections compared with baseline time, Denmark, 1 January 2010-31 December 2016

\begin{tabular}{|c|c|c|c|c|c|c|c|c|c|}
\hline \multirow[b]{2}{*}{$\begin{array}{l}\text { Days after sample } \\
\text { collection }^{\mathrm{a}}\end{array}$} & \multicolumn{3}{|c|}{ Streptococcus pneumoniae } & \multicolumn{3}{|c|}{ Respiratory viruses ${ }^{b}$ (combined) } & \multicolumn{3}{|c|}{ Influenza } \\
\hline & $\begin{array}{c}\text { Eventsc } \\
\text { (n) }\end{array}$ & IR (Cl 95\%) & $p$ value & $\begin{array}{c}\text { Eventsc } \\
\text { (n) }\end{array}$ & IR (CI 95\%) & $p$ value & $\begin{array}{l}\text { Events }^{c} \\
\text { (n) }\end{array}$ & IR (CI 95\%) & $p$ value \\
\hline \multicolumn{10}{|c|}{ Myocardial infarction } \\
\hline $1-3$ & 6 & $20.1(8.9-45.8)$ & $<0.001$ & 8 & $15.2(7.5-31.1)$ & $<0.001$ & 8 & $17.5(8.5-36.2)$ & $<0.001$ \\
\hline $4-7$ & 4 & $11.0(4.1-29.8)$ & $<0.001$ & 3 & $4.5(1.4-14.0)$ & 0.010 & 3 & $5.1(1.6-16.3)$ & 0.005 \\
\hline $8-14$ & 3 & $4.9(1.6-15.6)$ & 0.006 & 5 & $4.4(1.8-10.8)$ & 0.001 & 3 & $3.1(1.0-9.7)$ & 0.056 \\
\hline $15-28$ & 0 & ND & ND & 4 & $1.9(0.7-5.1)$ & 0.205 & 3 & $1.7(0.5-5.3)$ & 0.374 \\
\hline Baseline & Ref & 1 (ref) & NA & Ref & 1 (ref) & NA & Ref & 1 (ref) & NA \\
\hline \multicolumn{10}{|l|}{ Stroke } \\
\hline $1-3$ & 12 & $25.5(14.2-45.8)$ & $<0.001$ & 5 & $8.3(3.4-20.2)$ & $<0.001$ & 5 & $10.3(4.2-25.4)$ & $<0.001$ \\
\hline $4-7$ & 2 & $3.5(0.9-14.1)$ & 0.079 & 6 & $7.8(3.4-17.5)$ & $<0.001$ & 4 & $6.5(2.4-17.7)$ & $<0.001$ \\
\hline $8-14$ & 6 & $6.3(2.8-14.4)$ & $<0.001$ & 8 & $6.2(3.0-12.6)$ & $<0.001$ & 6 & $5.9(2.6-13.4)$ & $<0.001$ \\
\hline $15-28$ & 16 & $9.2(5.5-15.4)$ & $<0.001$ & 5 & $2.0(0.8-4.9)$ & 0.119 & 2 & $1.0(0.3-4.2)$ & 0.956 \\
\hline Baseline & Ref & 1 (ref) & NA & Ref & 1 (ref) & NA & Ref & 1 (ref) & NA \\
\hline
\end{tabular}

$\mathrm{Cl}$ : confidence interval; IR: incidence ratio; NA: not applicable; ND: could not be estimated (not determined); ref: reference.

a The sample collection time approximates the time of symptom onset of the respiratory infection.

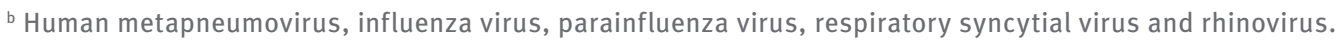

c Events of first acute myocardial infarction or stroke.

To investigate the potential effect modification of age on the association between respiratory infections and myocardial infarction/stroke, age was stratified on 40-64 years and $\geq 65$ years [7].

Data extraction and linking of data were performed using SAS version 9.4 [15]. STATA version 13 [16] was used for the SCCS analyses.

Ethical statement

No ethical approval was required for this registerbased study

\section{Results}

From 2000 to 2016, a total of 1,199 individuals with acute myocardial infarction and 1,205 individuals with stroke who also were diagnosed with either a S. pneumoniae or a viral respiratory infection were identified. Among these respectively, 581 with acute myocardial infarction (48\%) and 451 individuals with stroke (37\%), were excluded because their first acute myocardial infarction/stroke diagnosis occurred during the 10 year look-back period. This left 618 with acute myocardial infarction and 754 individuals with stroke who also had either a S. pneumoniae or a viral respiratory infection. When combining information on bacterial and viral respiratory infections, six individuals diagnosed with acute myocardial infarction and four diagnosed with stroke had both $S$. pneumoniae and a viral respiratory infection during the study period. Because the current study aimed to estimate the relative incidence of acute myocardial infarction and stroke separately for bacterial and viral infections, these individuals were excluded. Moreover, because the records with the two types of pathogens appeared on two different data files (i.e. bacterial or viral), these patients had each been counted twice among the respective 618 and 754 individuals with acute myocardial infection or stroke; this was accounted for when removing the records. In addition, two individuals were diagnosed with stroke after the date of death and excluded from the analysis. This left 606 individuals for the acute myocardial infarction analysis and 744 individuals for the stroke analysis (Figure 2 and Table 1 ).

Among the 606 individuals diagnosed with acute myocardial infarction, 628 respiratory infection episodes were detected. Of these, 406 infections were due to respiratory viruses with an average of 1.04 episodes per individual (range: 1-4 per individual), and 222 infections were due to $S$. pneumoniae with an average of 1.02 episodes per individual (range: $1-2$ per individual) (Table 1).

Among the 744 individuals diagnosed with stroke, a total of 766 laboratory-confirmed respiratory infections were detected during the study period. Of the 766 corresponding samples, 434 samples contained respiratory viruses with an average of 1.03 episodes per individual (range: 1-3 per individual), while 332 samples contained S. pneumoniae with an average of 1.03 episodes per individual (range: $1-3$ per individual) (Table 1).

All in all, among individuals with acute myocardial infarction the proportion of women was $41.7 \%$, while among individuals with stroke this was $46.6 \%$. Individuals diagnosed with acute myocardial infarction appeared to be younger with $34.7 \%$ (210/606) aged 40-64years compared with $28.8 \%$ (214/744) for 
Age and season adjusted incidence ratio for first acute myocardial infarction $(n=555)$ and first stroke $(n=656)$ after excluding patients who died within 30 days of Streptococcus pneumoniae and respiratory viruses infections (combined) compared with baseline time, Denmark, 1 January 2010-31 December 2016

\begin{tabular}{|c|c|c|c|c|}
\hline \multirow{2}{*}{ Days after sample collection ${ }^{a}$} & \multicolumn{2}{|c|}{ Streptococcus pneumoniae } & \multicolumn{2}{|c|}{ Respiratory viruses ${ }^{b}$ (combined) } \\
\hline & IR (CI 95\%) & $\mathrm{p}$ value & IR (Cl 95\%) & $\mathrm{p}$ value \\
\hline \multicolumn{5}{|l|}{ Myocardial infarction } \\
\hline $1-3$ & $18.2(7.4-44.6)$ & $<0.001$ & $14.8(6.9-31.6)$ & $<0.001$ \\
\hline $4-7$ & $11.4(4.2-30.9)$ & $<0.001$ & $4.9(1.6-15.4)$ & 0.006 \\
\hline $8-14$ & $3.4(0.8-13.6)$ & 0.090 & $3.9(1.4-10.4)$ & 0.008 \\
\hline $15-28$ & ND & ND & $2.0(0.8-5.5)$ & 0.157 \\
\hline Baseline & 1 (ref) & NA & 1 (ref) & $\mathrm{NA}$ \\
\hline \multicolumn{5}{|l|}{ Stroke } \\
\hline $1-3$ & $21.9(10.7-44.6)$ & $<0.001$ & $7.9(2.9-21.2)$ & $<0.001$ \\
\hline $4-7$ & $2.2(0.3-15.9)$ & 0.425 & $7.6(3.1-18.5)$ & $<0.001$ \\
\hline $8-14$ & $7.8(3.5-17.7)$ & $<0.001$ & $6.2(2.9-13.3)$ & $<0.001$ \\
\hline $15-28$ & $9.6(5.5-16.6)$ & $<0.001$ & $1.4(0.4-4.4)$ & 0.564 \\
\hline Baseline & 1 (ref) & NA & 1 (ref) & NA \\
\hline
\end{tabular}

$\mathrm{Cl}$ : confidence interval; IR: incidence ratio; NA: not applicable; ND: could not be estimated; ref: reference.

a The sample collection time approximates the time of symptom onset due to infection with either Streptococcus pneumoniae or respiratory viruses.

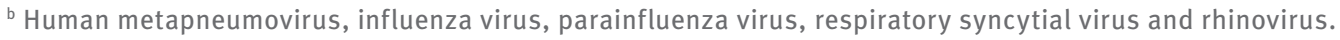

those with stroke. The observed mortality in this study, overall, and within 30 days post-cardiovascular event, seemed highest among stroke individuals $45.2 \%$ and $11.8 \%$, respectively, as compared with acute myocardial infarction individuals $39.9 \%$ and $8.4 \%$, respectively (Table 1).

In the age and season adjusted analyses for first acute myocardial infarction, the IRs were significantly elevated 1-14 days following infections with S. pneumoniae and respiratory viruses as compared with the baseline period (Table 2). The IRs for acute myocardial infarction following an $S$. pneumoniae infection were $20.1,11.0$ and 4.9 during $1-3,4-7$ and 8-14 days, respectively. The IRs for acute myocardial infarction following a respiratory virus infection were $15.2,4.5$ and 4.4 during $1-3,4-7$ and $8-14$ days, respectively.

In the age and season adjusted analyses for first stroke, the IRs of a stroke occurring during the risk period were significantly elevated 1-28days following infection with S. pneumoniae except for the time period 4-7 days. The IRs for stroke following an S. pneumoniae infection were $25.5,3.5,6.3$ and 9.2 during $1-3,4-7,8-14$ and 15-28 days, respectively. The IRs for stroke following a respiratory virus infection were significantly elevated 1-14 days and the IRs were 8.3, 7.8 and 6.2 during 1-3, 4-7 and 8-14 days, respectively (Table 2 ).

In the age and season adjusted sub-analyses for first acute myocardial infarction and stroke where only influenza virus infections were included, the estimated IRs were similar to the IRs obtained in the analysis including all respiratory virus infections (Table 2 ).
Excluding those who died within 30 days In total 51 (8.4\%) and 88 (11.8\%) individuals died within 30 days after acute myocardial infarction and stroke, respectively (Table 1). Excluding those who died within 30 days after an event provided results that were mostly unchanged from the main analysis (Table 3), although with slightly attenuated effect estimates. Using the risk period 1-3 days as an example, the acute myocardial infarction IR after S. pneumoniae infection changed from 20.1 to 18.2 and for respiratory viruses the IR changed from 15.2 to 14.8 . The stroke IR after S. pneumoniae infection changed from 25.5 to 21.9 and for respiratory virus the change was from 8.3 to 7.9 .

\section{Stratifying by age}

For both acute myocardial infarction and stroke, the number of Individuals aged between 40 and 64 years of age covered approximately one third of the total number of individuals included in the main analyses, for some risk periods no events were observed and IR could not be estimated. In addition, several IR estimates had wide confidence intervals. In particular the estimated IRs for the 1-3 days risk periods appeared to be higher in the 40-64years age stratified analyses compared with what we observed in the main analysis (Table 2). Following onset of an $S$. pneumoniae infection, the acute myocardial infarction IR was 37.1 and for stroke the IR was 43.3 during the 1-3 days risk period among 40-64year olds. In the 1-3 days after onset of a respiratory virus infection, the acute myocardial infarction IR was 5.1 and non-significant while during the same period the stroke IR was 16.4 and significant (Table 4). IRs for all risk periods are shown in Table 4. For the age group $\geq 65$ years the acute myocardial infarction and stroke IRs were similar to the IR estimates observed 
Age and season adjusted incidence ratio for first acute myocardial infarction $(n=606)$ and first stroke $(n=744)$ stratified by age $40-64$ years and $\geq 65$ years in periods after Streptococcus pneumoniae and respiratory viruses infections (combined) compared with baseline time, Denmark, 1 January 2010-31 December 2016

\begin{tabular}{|c|c|c|c|c|}
\hline \multirow{2}{*}{ Days after sample collection ${ }^{\mathrm{a}}$} & \multicolumn{2}{|c|}{ Streptococcus pneumoniae } & \multicolumn{2}{|c|}{ Respiratory viruses ${ }^{\mathrm{b}}$ (combined) } \\
\hline & IR (Cl 95\%) & $p$ value & $\mathrm{IR}(\mathrm{Cl}$ 95\%) & $p$ value \\
\hline \multicolumn{5}{|l|}{ Myocardial infarction } \\
\hline \multicolumn{5}{|l|}{$40-64$ years $(n=210)$} \\
\hline $1-3$ & $37.1(8.7-158.3)$ & $<0.001$ & $5.1(0.7-36.6)$ & 0.108 \\
\hline $4-7$ & $14.6(2.0-108.7)$ & 0.009 & $7.6(1.9-31.2)$ & 0.005 \\
\hline $8-14$ & ND & ND & $2.2(0.3-16.1)$ & 0.426 \\
\hline $15-28$ & ND & ND & $1.2(0.2-8.4)$ & 0.880 \\
\hline Baseline & 1 (ref) & NA & 1 (ref) & NA \\
\hline \multicolumn{5}{|l|}{$\geq 65$ years $(n=396)$} \\
\hline $1-3$ & $16.1(5.5-47.2)$ & $<0.001$ & $21.1(9.8-45.6)$ & $<0.001$ \\
\hline $4-7$ & $10.7(3.2-35.0)$ & $<0.001$ & $2.4(0.3-17.3)$ & 0.381 \\
\hline $8-14$ & $6.2(1.9-20.5)$ & 0.003 & $5.9(2.1-16.0)$ & 0.001 \\
\hline $15-28$ & ND & ND & $2.4(0.8-7.6)$ & 0.134 \\
\hline Baseline & 1 (ref) & NA & 1 (ref) & NA \\
\hline \multicolumn{5}{|l|}{ Stroke } \\
\hline \multicolumn{5}{|l|}{$40-64$ years $(n=214)$} \\
\hline $1-3$ & $43.3(15.3-122.5)$ & $<0.001$ & $16.4(5.1-52.89)$ & $<0.001$ \\
\hline $4-7$ & NA & NA & $8.4(2.0-34.7)$ & 0.003 \\
\hline $8-14$ & $10.3(2.5-43.2)$ & 0.001 & $10.4(3.7-28.9)$ & $<0.001$ \\
\hline $15-28$ & $14.1(5.5-36.1)$ & $<0.001$ & ND & ND \\
\hline Baseline & 1 (ref) & NA & 1 (ref) & NA \\
\hline \multicolumn{5}{|l|}{$\geq 65$ years $(n=530)$} \\
\hline $1-3$ & $20.6(10.1-42.3)$ & $<0.001$ & $4.9(1.2-20.0)$ & 0.025 \\
\hline $4-7$ & $4.3(1.1-17.4)$ & 0.041 & $7.7(2.9-21.0)$ & $<0.001$ \\
\hline $8-14$ & $5.3(1.9-14.2)$ & 0.001 & $4.6(1.7-12.5)$ & 0.003 \\
\hline $15-28$ & $7.9(4.2-14.7)$ & $<0.001$ & $3.0(1.2-7.4)$ & 0.016 \\
\hline Baseline & 1 (ref) & NA & 1 (ref) & NA \\
\hline
\end{tabular}

$\mathrm{Cl}$ : confidence interval; IR: incidence ratio; NA: not applicable; ND: could not be estimated (no events); ref: reference.

a The sample collection time approximates the time of symptom onset due to infection with either Streptococcus pneumoniae or respiratory viruses.

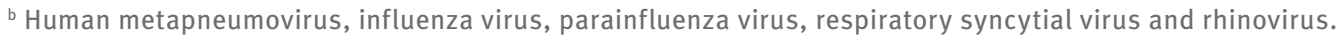

in the main analyses. Acute myocardial infarction and stroke IRs for all risk periods are shown in Table 4.

\section{Discussion}

We found that the IRs of first acute myocardial infarction and stroke were markedly elevated following infections with S. pneumoniae and selected respiratory viruses (mainly influenza virus) compared with baseline time periods using national linked records from Denmark. The elevated IRs were observed during the risk periods 1-14 days following infection, except for the stroke IRs following S. pneumoniae infection where an elevated IR was observed during the whole risk period apart from the 4-7 day period.

The IR patterns observed during the risk periods in our study were similar to the patterns observed in the Scottish and Canadian studies with the highest IRs observed 1-3 days after infection followed by a decrease in IRs with increasing time since infection
$[5,7]$. The estimated IRs were in general higher in our study compared with the Scottish and Canadian studies in particular during the risk window 1-3 days following infection. The IRs for acute myocardial infarction following S. pneumoniae and respiratory virus infections in the Scottish study were 5.98 and 5.59 , respectively 1-3 days following infection, compared with 20.1 and 15.2 in our study. The IRs for acute myocardial infarction 1-3 days following influenza infection were 9.8 in the Scottish study [7], 6.3 in the Canadian study [5] and 17.5 in our study. The IR for stroke in the Scottish study was 12.3 following $S$. pneumoniae infection and 6.79 following infection with respiratory viruses during the 1-3 days risk window. In our study, the corresponding IRs were 25.5 and 8.3. In the Scottish study, acute myocardial infarction and stroke IRs were higher following influenza infections compared with other respiratory viruses. In our study, the sample size of other viruses than influenza only accounted for $20 \%$ of all respiratory viruses; therefore, due to the small 
sample size, it was not possible to make a similar comparison based on Danish data.

In the Scottish study, the estimated IRs for the effect of respiratory viruses were generally higher among individuals aged 40-64years compared with those aged $\geq 65$ years, and this was suggested to possibly be due to a higher influenza vaccine coverage among those aged $\geq 65$ years [7]. In our study, the IRs associated with respiratory viruses were not in general higher among individuals below 65 years old, although confidence intervals were wide. However, the IRs for myocardial infarction and stroke 1-3 days following S. pneumoniae infection in the age group 40-64 years were more than double those of the age group $\geq 65$ years. Based on the assumption that the pneumococcal vaccine may reduce the risk of cardiac outcomes, either by preventing infection or lessening its severity, this might indicate that individuals aged $\geq 65$ years are aware of getting their recommended pneumococcal vaccine. A similar pattern was observed for stroke in the Scottish study [7]. However, the differences between the age strata observed in the Scottish study and ours require further investigation and it would be interesting to repeat the analysis with a larger sample size stratifying on influenza and $S$. pneumoniae vaccination status. Additionally analyses with stratifications based on influenza and pneumococcal subtypes, as well as taking into account the underlying comorbidities individuals may have, may provide knowledge allowing to further customise/target the influenza and pneumococcal vaccination programmes.

Acute myocardial infarction and stroke are related to a high mortality rate, which can result in a shortening of the observation period leading to bias in either direction [12]. In both the Scottish [7] and in our study, the estimated IRs when excluding those who died within 30 days after the event produced results similar to the main analysis indicating that the high mortality did not bias the results (Table 2 and 3 ).

Individuals $\geq 65$ years and people at any age diagnosed with cardiovascular risk factors where an influenza infection can result in severe health effects can get the influenza vaccine for free in Denmark. However, they might not be aware that they have increased risk of acute myocardial infarction and stroke following an influenza infection. Genetic changes or differences in the circulating influenza viruses compared with the vaccine strains in some seasons can result in low or suboptimal vaccine effectiveness [17], however, in these seasons improvement of influenza vaccine coverage will still increase number of prevented influenza cases. S. pneumoniae is also vaccine preventable and the vaccine is recommended to individuals $\geq 65$ years, but in Denmark only individuals with very high risk of invasive pneumococcal disease (IPD) are covered by limited subsidy when vaccinated [18]. However there are other groups with increased risk of IPD where pneumococcal vaccination is recommended but not free of charge e.g. all individuals $\geq 65$ years of age who do not have an IPD risk classified as very high $[19,20]$; individuals of all ages diagnosed with chronic conditions such as heart disease and lung disease [21,22].

Strengths of our study include (i) use of SCCS analysis which implicitly controls for fixed between-person confounding effects and is statistically efficient compared with a cohort design, (ii) use of laboratory-confirmed infections from a national microbiology database combined with ICD-10 coded cardiovascular outcomes, which are shown to have high positive predictive values in validation studies from Denmark [23-25] that enhance confidence in the validity of measurements, (iii) use of national linked data across all of Denmark and (iv) use of the same protocol as the Scottish study to allow for direct comparisons between the studies. Although our study period was shorter and the sample size smaller than the Scottish one, the main results obtained by the two studies are comparable.

Our study also has limitations. With a sample size of 606 individuals in the acute myocardial infarction analyses, and 744 in the stroke analyses, totalling 1,350 individuals our study was smaller than the Scottish study [7], which resulted in lack of power in some sub-group analyses. In addition, there were too few non-influenza respiratory viruses to separate the respiratory virus analyses according to influenza vs noninfluenza viruses, and the limited sample size and the short duration of the time series did not enable us to disentangle influenza subtypes effects. Only very few individuals experienced co-infections and the effect of co-infections could not be investigated separately. For the respiratory infections we used date of sampling and not date of symptom onset, which means that the true date of infection occurred during the 14 days excluded from the analyses. The IRs decreased as time since sampling increased. The fact that we use date of sampling, which is after the date of infection, may lead to underestimation of the effect. The decision to swab an individual to verify a respiratory infection might be more likely for individuals with a severe infection or underlying risk factors for severe disease, which might indicate that the results from this study apply to more severe infections and/or vulnerable patients [5].

In conclusion, this study suggested a significant cardiovascular event triggering effect following infection with S. pneumoniae and respiratory viruses (mainly influenza). This was found not only in the age group $\geq 65$ years, but also among 40-64 year olds. The results were similar to those found in a Scottish population using the same study protocol, suggesting that they are generalisable across different northern European populations. These findings indicate the importance to inform general practitioners and individuals at risk of cardiovascular diseases about the importance of being protected against vaccine-preventable respiratory infections. 


\section{Acknowledgements}

The microbiological test results were obtained from the Danish Microbiology Database (MiBa, http://miba.ssi. $\mathrm{dk}$ ), which contains all electronic reports from departments of clinical microbiology in Denmark since 2010, and we acknowledge the collaboration with the MiBa Board of Representatives.

We also acknowledge Tine Dalby who is taking part in the national surveillance of invasive pneumococcal disease at SSI.

Funding statement: This work was funded by an Academy of Medical Sciences Starter Grant to Clinical Lecturers to CWG. CWG is supported by a Wellcome Intermediate Clinical Fellowship (201440_Z_16_Z). RMB is supported by a UKRI Innovation Fellowship funded by the Medical Research Council, UK (MR/So03797/1)

\section{Conflict of interest}

None declared.

\section{Authors' contributions}

Jessica Ohland was responsible for the SCCS analysis and led the writing of the paper together with Hanne-Dorthe Emborg. Hanne-Dorthe Emborg was responsible data extraction, validation and linking of data. Charlotte Warren-Gash had the initial idea to perform the study, together with Kåre Mølbak she conceptualised the study, and they discussed the data and perspectives together with Jessica Ohland, Hanne-Dorthe Emborg and Palle Valentiner-Branth. Ruth Blackburn and Jens Nielsen provided statistical input. All authors provided contributions to the paper and approved the final version.

\section{References}

1. World Health Organization (WHO). The top 10 causes of death. Geneva: WHO; 2018. [Accessed 26 Nov 2018]. Available from: www.who.int/mediacentre/factsheets/fs310/en/index.html

2. Collins SD. Excess Mortality from Causes Other than Influenza and Pneumonia during Influenza Epidemics. Public Health Rep. 1932;47(46):2159-79. https://doi.org/10.2307/4580606 PMID: 19315373

3. Warren-Gash C, Smeeth L, Hayward AC. Influenza as a trigger for acute myocardial infarction or death from cardiovascular disease: a systematic review. Lancet Infect Dis. 2009;9(10):601-10. https://doi.org/10.1016/S14733099(09)70233-6 PMID: 19778762

4. Barnes M, Heywood AE, Mahimbo A, Rahman B, Newall AT, Macintyre CR. Acute myocardial infarction and influenza: a meta-analysis of case-control studies. Heart. 2015;101(21):1738-47. https://doi.org/10.1136/ heartjnl-2015-307691 PMID: 26310262

5. Kwong JC, Schwartz KL, Campitelli MA, Chung H, Crowcroft NS, Karnauchow T, et al. Acute Myocardial Infarction after Laboratory-Confirmed Influenza Infection. N Engl J Med. 2018;378(26):345-53. https://doi.org/10.1056/NEJMoa1702090 PMID: 29949484

6. Blackburn R, Zhao H, Pebody R, Hayward A, Warren-Gash C. Laboratory-Confirmed Respiratory Infections as Predictors of Hospital Admission for Myocardial Infarction and Stroke: TimeSeries Analysis of English Data for 2004-2015. Clin Infect Dis. 2018;67(1):8-17. https://doi.org/10.1093/cid/cix1144 PMID: 29324996

7. Warren-Gash C, Blackburn R, Whitaker H, McMenamin J, Hayward AC. Laboratory-confirmed respiratory infections as triggers for acute myocardial infarction and stroke: a selfcontrolled case series analysis of national linked datasets from Scotland. Eur Respir J. 2018;51(3):1701794. https://doi. org/10.1183/13993003.01794-2017 PMID: 29563170
8. Corrales-Medina VF, Suh KN, Rose G, Chirinos JA, Doucette S, Cameron DW, et al. Cardiac complications in patients with community-acquired pneumonia: a systematic review and meta-analysis of observational studies. PLoS Med. 2011;8(6):e1001048. https://doi.org/10.1371/journal. pmed.1001048 PMID: 21738449

9. Mazur NI, Higgins D, Nunes MC, Melero JA, Langedijk AC, Horsley N, et al. Respiratory Syncytial Virus Network (ReSViNET) Foundation. The respiratory syncytial virus vaccine landscape: lessons from the graveyard and promising candidates. Lancet Infect Dis. 2018;18(10):e295-311. https:// doi.org/10.1016/S1473-3099(18)30292-5 PMID: 29914800

10. Voldstedlund M, Haarh M, Mølbak KMiBa Board of Representatives. The Danish Microbiology Database (MiBa) 2010 to 2013. Euro Surveill. 2014;19(1):20667. https://doi. org/10.2807/1560-7917.ES2014.19.1.20667 PMID: 24434175

11. Whitaker HJ, Farrington CP, Spiessens B, Musonda P. Tutorial in biostatistics: the self-controlled case series method. Stat Med. 2006;25(10):1768-97. https://doi.org/10.1002/sim.2302 PMID: 16220518

12. Petersen I, Douglas I, Whitaker H. Self controlled case series methods: an alternative to standard epidemiological study designs. BMJ. 2016;354:i4515. https://doi.org/10.1136/bmj. i 4515 PMID: 27618829

13. Andersen TF, Madsen M, Jørgensen J, Mellemkjoer L, Olsen JH. The Danish National Hospital Register. A valuable source of data for modern health sciences. Dan Med Bull. 1999;46(3):263-8. PMID: 10421985

14. World Health Organization (WHO). International Statistical Classification of Diseases and Health-related Problems, 10th Revision. Geneva: WHO; 2016. Available from: http://apps.who. int/classifications/icd10/browse/2016/en

15. SAS. SAS. Cary, NC, USA: SAS Institute.

16. StataCorp. Stata Statistical Software. College Station, TX: StataCorp LP.

17. Trebbien R, Fischer TK, Krause TG, Nielsen L, Nielsen XC, Weinreich LS, et al. Changes in genetically drifted $\mathrm{H}_{3} \mathrm{~N}_{2}$ influenza A viruses and vaccine effectiveness in adults 65 years and older during the 2016/17 season in Denmark. J Clin Virol. 2017;94:1-7. https://doi.org/10.1016/j.jcv.2017.06.007 PMID: 28697450

18. Kants $\varnothing$ B, Jørgensen CS, Slotved HC, Hoffman S, Suppli CH, Valentiner-Branth P. Forslag til pneumokokvaccination uden for børnevaccinationsprogrammet. [Suggestions for pneumococcal vaccination outside the childhood vaccination programme]. Copenhagen: Statens Serum Institut; 2014.

19. Kantsø B. Pneumokokvaccination uden for børnevaccinationsprogrammet i Danmark. [Pneumococcal vaccination outside the childhood vaccination programme in Denmark]. Report No.: Version 1.3. Copenhagen: Statens Serum Institut; 2014. p. 1-30.

20. Harboe ZB, Benfield TL, Valentiner-Branth P, Hjuler T, Lambertsen L, Kaltoft M, et al. Temporal trends in invasive pneumococcal disease and pneumococcal serotypes over 7 decades. Clin Infect Dis. 2010;50(3):329-37. https://doi. org/10.1086/649872 PMID: 20047478

21. Kyaw MH, Rose CE Jr, Fry AM, Singleton JA, Moore Z, Zell ER, et al. Active Bacterial Core Surveillance Program of the Emerging Infections Program Network. The influence of chronic illnesses on the incidence of invasive pneumococcal disease in adults. I Infect Dis. 2005;192(3):377-86. https://doi.org/10.1086/431521 PMID: 15995950

22. van Hoek AJ, Andrews N, Waight PA, Stowe J, Gates P, George R, et al. The effect of underlying clinical conditions on the risk of developing invasive pneumococcal disease in England. J Infect. 2012;65(1):17-24. https://doi.org/10.1016/j. jinf.2012.02.017 PMID: 22394683

23. Sundbøll J, Adelborg K, Munch T, Frøslev T, Sørensen HT, Bøtker HE, et al. Positive predictive value of cardiovascular diagnoses in the Danish National Patient Registry: a validation study. BMJ Open. 2016;6(11):e012832. https://doi.org/10.1136/ bmjopen-2016-012832 PMID: 27864249

24. Krarup L-H, Boysen G, Janjua H, Prescott E, Truelsen T. Validity of stroke diagnoses in a National Register of Patients. Neuroepidemiology. 2007;28(3):150-4. https://doi. org/10.1159/000102143 PMID: 17478969

25. Johnsen SP, Overvad K, Sørensen HT, Tjønneland A, Husted $\mathrm{SE}$. Predictive value of stroke and transient ischemic attack discharge diagnoses in The Danish National Registry of Patients. J Clin Epidemiol. 2002;55(6):602-7. https://doi. org/10.1016/S0895-4356(02)00391-8 PMID: 12063102 
License, supplementary material and copyright

This is an open-access article distributed under the terms of the Creative Commons Attribution (CC BY 4.0) Licence. You may share and adapt the material, but must give appropriate credit to the source, provide a link to the licence and indicate if changes were made.

Any supplementary material referenced in the article can be found in the online version.

This article is copyright of the authors or their affiliated institutions, 2020. 